\title{
The Analysis of the Preventive Nursing of Infection after Orthopedic Operation
}

\author{
$\operatorname{Min} \mathbf{A i}^{*}$ \\ The General Hospital of Shuicheng Goal Mine Industry Group, Liupanshui, Guizhou 553000, China
}

\begin{abstract}
Objective: To explore the preventive nursing methods of infection after orthopedic operation and provide some basis for clinical nursing. Method: $1000 \mathrm{pa}-$ tients who have received orthopedic operations are selected randomly from our hospital from September 2009 to September 2014, then we make follow-up and survey, analyze the factors influencing infection and implement corresponding nursing measures. Result: Among the 1000 patients, 26 patients have incision infection of sterile operation and the infected positions are on spinal cord, upper limbs and lower limbs. Surveys find that after orthopedic operation, infection patients are mostly infected 3-4 weeks after being hospitalized with the earliest infection occurring after one week after being hospitalized. We can find that the longer patients are hospitalized for, the higher the occurrence of infection is. Conclusion: In the preventive nursing of infection after orthopedic operation, sterile operation and preoperative, intraoperative and postoperative nursing should be remembered. And the most important one is to adopt sterilization and isolation system after orthopedic operation to lower the occurrence of infection to the lowest.
\end{abstract}

\section{KEYWORDS}

Orthopedic operation

Infection

Preventive nursing

\section{Introduction}

As the constant development of modern society and science and technology, the occurrence of vehicle accidents constantly rises following the constant upgrading of human's transportation means. Many patients injured in vehicle accidents need to receive orthopedic operation as whether the preventive nursing of infection after orthopedic operation can be done properly will influence the clinical healing of patients. The common complication after orthopedic operation is incision infection. Incision infection after orthopedic operation can delay the healing of wounds and even cause osteomyelitis in the serious case. Therefore, after orthopedic operation, controlling incision infection is particularly important. By following and surveying the 1000 patients of our hospital who have received orthopedic operation from September 2009 to September 2014, the

Copyright ๑ $2015 \mathrm{Min} \mathrm{Ai}$

doi: $10.18686 /$ jn.v4i1.3

Received: December 4, 2014; Accepted: January 21, 2015; Published online: March 27, 2015

This is an open-access article distributed under the terms of the Creative Commons Attribution Unported License (http://creativecommons.org/ licenses/by-nc/4.0/), which permits unrestricted use, distribution, and reproduction in any medium, provided the original work is properly cited.

${ }^{\star}$ Corresponding author: The General Hospital of Shuicheng Goal Mine Industry Group, Liupanshui, Guizhou 553000, China. E-mail: am_ shuicheng@126.com paper explores the preventive nursing methods of infection after orthopedic operation to provide some basis for clinical nursing.

\section{Clinical data}

\subsection{Basic data}

The 1000 patients, having received orthopedic operation, randomly selected from our hospital from September 2009 to September 2014 include 654 males and 336 females. They age from $10-74$ and $(45 \pm 3.6)$ on average. Among the 1000 patients, 223 patients have received spinal operation, 604 patients have received limbs operation and 173 patients have received other operations.

\subsection{Diagnosis criteria}

The infection diagnosis criteria in the survey are mainly made by referring to Infection Diagnosis Criteria in Hospital [1] issued by Ministry of Health of China in 2001.

\subsection{Survey methods}

The paper tracks and nurses the 1,000 patients, also records the complications occurring after treatment, the nursing methods and curative effects.

\section{Result}

In the survey, 26 patients of 1,000 patients receiving ortho- 
pedic operation have operative incision infection with the infection rate of $2.6 \%$, of which there are 7 patients with infection in hip and femur, accounting for $26.92 \%$ of all infection patients, 12 with infection on spinal accounting for $46.15 \%$, 4 with infection on upper limbs accounting for $15.38 \%$ and 3 with infection on ankle accounting for $11.53 \%$. We can know from the above result that among operative incision infection, infection mainly occurs on hip, femur, spinal cord, upper limbs and ankle. 4 patients are infected 4 weeks after being hospitalized accounting for $15.38 \%$ of all infection patients; 19 patients are infected in the third and fourth week of hospitalization accounting for $73.07 \%$; and 3 patients are infected within two weeks after being hospitalized accounting for $11.53 \%$ of all infection patients. We can find from the above result that the longer patients are hospitalized for, the higher the occurrence of infection is. The experiment also finds that 24 infection patients are more than 55 years old, accounting for $92.3 \%$ of all infection patients.

\section{Analysis of infection causes}

Incision infection of orthopedic operation is caused by many factors including patient factors, iatrogenic factors and so on. But the author thinks correct preoperative and postoperative preventive nursing is one important factor preventing postoperative infection. (1) Patients themselves. Many patients judge whether operation is successful at the ending of operation and attribute completely the effects of operation to medical technologies and service abilities, in fact, the body adjustment and behaviors of patients before and after operation are also very important. (2) Nursing personnel should conduct nursing prevention according to the principle of specialty, service and pertinence and timely track the healing situation of postoperative wounds to lower the occurrence of postoperative infection furthest. (3) Hospital ignores the influences of the clean and neat department environment on the healing of postoperative wounds. The mixture of the normal bacteria inside patients' skin and body, and the highly toxic and drug-fast bacteria adds difficulties to the prevention and treatment of postoperative infection [2].

\section{Nursing}

Infection after orthopedic operation is a kind of complication and often occurs in the third and the fourth week after operation. The preventive nursing of infection is a kind of complex and sustainable work. Infection is caused by many factors, so in the preventive nursing after orthopedic operation, some measures should be adopted to avoid the factors which may cause infection [3].

\subsection{Preoperative nursing}

Nursing should be offered before orthopedic operation to make preparation for the subsequent nursing work. In the nursing before operation, physical examination, skin cleaning and the preparation of apparatuses in operation should be finished.

\subsubsection{Preoperative physical examination}

Before operation, medical personnel should urge patients to receive strict physical examination in case that some diseases influence the curative effects of operation or other complications occur. Besides, medical personnel should also observe the medical records of patients in order to avoid the above situation and effectively prevent medical disputes. Meanwhile, senior patients or the patients with weak physical condition should be offered appropriate nutrition support.

\subsubsection{Skin cleaning}

In the skin cleaning before operation, operative site and its neighborhood should be cleaned absolutely.

\subsubsection{Preoperative apparatuses preparation and skin preparation}

Equipment should be prepared and sterilized before operation. Skin preparation should be conducted on the day of operation. When eliminating the hair on operation site, the method not wounding skin should be used, and blades should be avoided in case of postoperative infection [4].

\subsection{Intraoperative nursing}

In operation, the monitoring in all directions should be done in case of different danger factors. Meanwhile, surgeon should improve its operation skills and master skillfully different technologies in operation. In operation, surgeon should avoid leaving residual cavity when sewing and ensure full drainage. And nurses should get familiar with different operation equipment and in operation cooperate with surgeon tacitly to reduce the time of operation and lower the occurrence of postoperative infection furthest. Personnel flow should be strictly controlled in operation. Except for necessary personnel, the number of personnel should be lowered furthest in operation room in case of bacteria spreading [5].

\subsection{Postoperative nursing 5.3.1. Conditions observation}

Nursing personnel should pay attention to drain blood and wound discharge. When dressing is founded to be contaminated or falls, it should be replaced timely. Nursing personnel should pay attention to the blood circulation of diseased limbs, closely observe the temperature, color and swelling of limb end and make relevant records; nursing personnel should pay attention to the pains of patients, ask patients' tolerance and pains nature and judge whether incision infection exists according to results. Once continuous jumping pain and body temperature rise are found, incision infection should be considered, the charge doctor should be informed, and antibiotic [6] and other corre- 
sponding treatment should be offered.

\subsubsection{Drugs replacing nursing}

The drugs replacing room should have a clean drugs replacing environment and should not allow other personnel in. When replacing drugs, medical personnel should wash hands, keep bacteria away from operation, use onetime mask, keep drugs replacing room clean, keep personnel from walking in the room, and appropriately bind up wound in case of falling.

\subsubsection{Nutrition support}

The nutritional state of whole body is closely related to the occurrence of postoperative incision infection. Therefore, for fracture patients, especially senior patients, nutrition support treatment should eat more high protein and high vitamin food, including vegetables and fruits, to keep defecation unobstructed, and when necessary, offer intravenous nutrition support to strengthen the immunity of body.

\subsubsection{Ward environment}

In case of the influences of excessively low temperature on the blood supply of tissues in diseased part, the temperature in wards in winter should be kept at $15-18{ }^{\circ} \mathrm{C}$ and the temperature in severed finger reunion operation room should be kept at $2-5{ }^{\circ} \mathrm{C}$; the temperature in rooms in summer should be controlled to make patients sweat a little or feel cool and keep wounds dry to prevent infection of wounds caused by soaking in sweat. Wards should be mopped with soda water. Nursing personnel should guide patients to turn over and do other bed activities without touching operation incision. The protection work of incision should be done when coughing disappears.

\section{Conclusion}

The commonest incision infection in orthopedic operation is bacteria infection, and the widest clinically used drugs in preventing incision infection is antibiotics. Incision infection after orthopedic operation is caused by many factors including patient factors and iatrogenic factors [7]. One research finds [8] that there is no obvious difference between using antibiotics and not using antibiotic in preventing incision infection, but the long-term use of antibiotic can make infection rise, make bacteria have drug resistance, improve the occurrence of field plating of drug-resistant bacteria and maybe make drug-resistant bacteria spread in endemic area and prevail in regions. The survey finds that hip and femur operations have the highest occurrence of infection mainly because the incision is deeper in the operations and they are complex, which requires incision to be exposed to air for longer time. Besides, we also find that the patients being hospitalized for 3-4 weeks are easiest infected mainly because there are many bacteria on skin surface and in incision. Therefore, the nursing work mentioned in this paper should be done in the postoperative preventive nursing in order to provide the reliable technical guarantee for the healing of patients.

\section{References}

1. Wu J, Zeng $F$. The preventive nursing of after orthopedic operation. Nursing Garden. 2013;10(24):693-695.

2. The Guidelines of Prevention and Control Technologies of Site Infection after Surgical Operations (pilot test) issued by Ministry of Health of PRC (WBYF [2010] No. 187). Beijing:Ministry of Health of PRC. 2010.

3. Fan $X$. The prevention and control of risk factors of pulmonary infection of senior hip and fracture patients in hospital. Chinese Journal of Nosocomiology. 2009;19(9):1055-1057.

4. Yang $X$. The preventive nursing of infection after orthopedic operation. Nei Mongol Journal of Traditional Chinese Medicine. 2013;32(4):169-170.

5. Lu Y, Lv ZQ. The analysis of preventing nursing measures of infection after orthopedic operation. China Health Industry. 2012(12):37-38.

6. Luo $\mathrm{H}$. The preventive nursing of incision infection and the application of antibiotics. Chinese Journal of Modern Drug Application. 2009;17(3):173.

7. Lu H, Zhang ZW, Xu HW. The analysis of relevant factors of incision infection in hospital after orthopedic operation. Chinese Journal of Nosocomiology. 2011;21(8):1548-1550.

8. Yan Z, Yang XY, Mai W. Causes analysis and solutions of 182 patients infected in hospital after orthopedic operation. Chinese Journal of Nosocomiology. 2005;15(2):155157. 